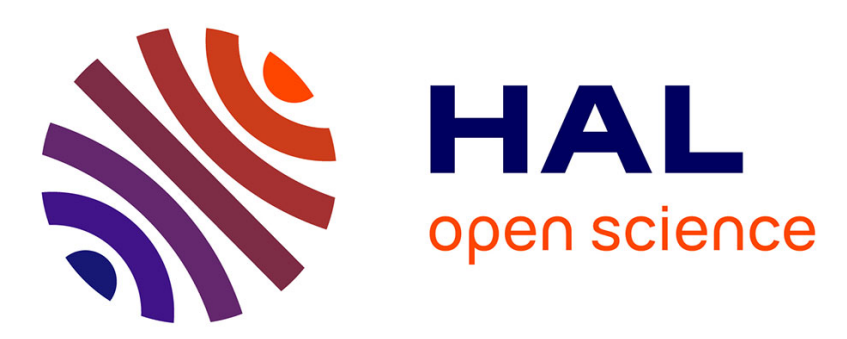

\title{
Se sentir bien dans chacun des lieux de l'espace scolaire au collège
}

\author{
Isabelle Joing, Olivier Vors, Clement Llena, François Potdevin
}

\section{To cite this version:}

Isabelle Joing, Olivier Vors, Clement Llena, François Potdevin. Se sentir bien dans chacun des lieux de l'espace scolaire au collège. Spiral-E (Supplément), 2018, Spiral-E, 61, pp.19-40. hal-02534630

\section{HAL Id: hal-02534630 \\ https://hal.univ-lille.fr/hal-02534630}

Submitted on 7 Apr 2020

HAL is a multi-disciplinary open access archive for the deposit and dissemination of scientific research documents, whether they are published or not. The documents may come from teaching and research institutions in France or abroad, or from public or private research centers.
L'archive ouverte pluridisciplinaire HAL, est destinée au dépôt et à la diffusion de documents scientifiques de niveau recherche, publiés ou non, émanant des établissements d'enseignement et de recherche français ou étrangers, des laboratoires publics ou privés. 


\title{
SE SENTIR BIEN DANS CHACUN DES LIEUX DE L'ESPACE SCOLAIRE AU COLLÈGE
}

\author{
LE RÔLE DE L'AUTONOMIE, \\ DE L'APPARTENANCE SOCIALE, \\ DE LA PERCEPTION DU LIEU \\ ET DU SENTIMENT DE SÉCURITÉ
}

\begin{abstract}
Résumé : L'objet de ce travail est d'examiner et d'expliquer la variation des expériences vécues au collège (composante affective du bien-être subjectif) en fonction des lieux fréquentés par l'élève. De nombreuses recherches questionnent le bien-être en contexte scolaire de manière globale sans distinguer nécessairement les différents lieux de l'espace scolaire. La présente recherche postule que le bien-être au collège varie quotidiennement en fonction des lieux fréquentés par l'élève et que cette variation peut s'expliquer par le degré de satisfaction des besoins psychologiques et/ou physiologiques dans chacun des lieux, par la perception physique du lieu ou encore par le climat qui y règne. L'enquête de terrain a été menée auprès de 2028 élèves issus de dix collèges publics des Hauts-deFrance. Après avoir identifié les lieux pour lesquels les élèves déclarent vivre le plus d'expériences positives (à savoir la cour de récréation, la cantine, la salle de sport et le CDI) et ceux pour lesquels les scores de bien-être affectif sont significativement bas (les lieux institutionnels tels que l'administration, le bureau de la vie scolaire ou du CPE, ainsi que les toilettes), les résultats révèlent que la qualité des expériences vécues dans un lieu est conditionnée par le fait de vivre des activités qui contribuent à la satisfaction des besoins d'affiliation et d'autonomie, de percevoir le lieu comme agréable, accueillant et propre, de ne pas être victime d'incivilités par les pairs, et de se sentir en sécurité.

Mots-clefs : espaces, collège, bien-être affectif, affiliation, victimation, école, cli-
\end{abstract} mat scolaire.

\section{INRODUCTION}

Etudier le bien-être des enfants à l'école apparaît essentiel au regard du temps passé au sein de cette institution (Hui \& Sun, 2010). Leur satisfaction de vie globale et leur développement personnel semblent en effet largement impactés par les expériences qui y sont vécues (Huebner, 1994 pour les investigations précoces sur le sujet ; Baker, Dilly, Aupperlee \& Patil, 2003 ; Danielsen, Samdal, Hetland \& Wold, 2009). Pour autant, cette préoccupation est relativement récente dans la littérature scientifique en France (Lenoir, 2012 ; Ferrière et al., 2016). D’un point de vue institutionnel, la question du bien-être des élèves est peu considérée ; les textes officiels français relatant très peu cette thématique (Florin \& Guimard, 2017 ; Nguyen, 2016).

Spiral-E - Revue de Recherches en Éducation - 2018

Supplément électronique au $N^{\circ} 61$ (19-40) 
Dans cette recherche, nous souhaitons nous intéresser plus particulièrement à la scolarité au collège, période sensible de l'adolescence durant laquelle le niveau de bien-être global de l'élève a tendance à diminuer (Gonzalez-Carrasco et al., 2016 ; Guimard et al., 2017).

L'entrée au collège est associée à un changement d'organisation (plusieurs salles de classe, nouveaux espaces de fréquentation, sonneries rythmant la journée scolaire...) qui font que l'élève côtoie désormais une diversité de lieux et de référents éducatifs plus importante qu'à l'école primaire (Derouet-Besson, 2005). Sur ce point, Eccles et ses collaborateurs (Eccles \& Midgley, 1989; Eccles et al., 1984 ; Eccles et al., 1993) affirment que le changement d'environnement éducatif associé à l'entrée au collège peut être une explication possible au déclin de l'engagement scolaire.

Pourtant, lorsque le bien-être est questionné dans la littérature, la question d'espaces est quasiment absente de la réflexion ; le bien-être de l'élève est interrogé de manière globale à l'échelle de l'établissement, ou à l'échelle de la classe la plupart du temps. Deux questions animent alors ce travail et définissent son objet : la qualité des expériences vécues par les élèves est-elle la même quel que soit le lieu fréquenté dans le collège ? Comment expliquer qu'un élève se sente particulièrement bien dans un lieu et moins bien dans un autre?

Bien-être subjectif :

éclairages théoriques

Le bien-être subjectif peut être éclairé de plusieurs manières. Tout d'abord il convient de souligner les nombreuses différences inter-individuelles : les sentiments de bonheur et de satisfaction diffèrent fortement selon les individus (Myers, 1992 ; Ryff, 1995 ; Waterman, 1993) mais aussi selon les contextes culturels (Diener et al., 1995). Sur le plan individuel, une des manières d'étudier le concept consiste à considérer que le bien-être de chacun varie d'un jour à l'autre et d'un environnement à l'autre (Reis et al., 2000) et qu'il ne constitue donc pas une donnée stable.

En accord avec cette logique, Diener (1996) a noté la nécessité d'améliorer la psychologie bien établie du bien-être autodéclaré avec des comptes rendus d'influences situationnelles et circonstancielles. C'est pourquoi, dans ce travail, nous nous intéresserons notamment à la qualité des expériences (positives ou négatives) qu'un élève vit dans les différents lieux de l'espace scolaire (composante affective du bien-être subjectif). Selon Diener (1984, 1994), un sentiment positif de bienêtre subjectif consiste à vivre beaucoup d'affects positifs, peu d'affects désagréables et à ressentir une grande satisfaction générale à l'égard de sa vie. Appliquée au bien-être des élèves à l'école, cette approche hédonique du bien-être revient à évaluer la qualité des expériences qu'ils vivent dans ce contexte et à évaluer le niveau de satisfaction qu'ils ont à y séjourner. Pour ce travail, nous ne retiendrons que la première dimension (dimension affective).

S'intéresser au bien-être affectif, c'est également mettre en avant l'importance d'interroger directement l'élève afin de mieux comprendre ce qu'il vit dans chacun des lieux qu'il fréquente et in fine améliorer la qualité de vie à l'école (Elmore \& Huebner, 2010 ; Florin \& Guimard, 2017). Cela permet également de s'attacher à la mesure des états émotionnels momentanés vécus par l'individu. Cette dimension qui s'intéresse principalement aux émotions et aux humeurs positives et négatives relève de l'instantané, du « ici et maintenant », approche particulièrement adaptée à notre objet d'étude dont l'hypothèse centrale est que le bienêtre de l'élève varie en fonction des lieux fréquentés. 
Le collège : une diversité de lieux

qui ne sont pas neutres pour l'élève

La littérature sur le bien-être scolaire fonctionne comme si l'établissement scolaire était un lieu unique et homogène constitué par les salles de classe principalement. Pourtant, si l'établissement peut constituer une unité de fonctionnement, l'espace scolaire se compose de différents lieux. La salle de cours mais aussi le hall d'entrée, la cours de récréation, le restaurant scolaire, les couloirs, les vestiaires d'Éducation Physique et Sportive (EPS), le Centre de Documentation et d'Information (CDI) ${ }^{1}$ ou encore l'arrêt de bus de l'établissement sont autant de lieux fréquentés par les élèves et dédiés aux différentes formes d'apprentissage des savoirs et de socialisation de celles et ceux qui les fréquentent (Mazalto \& Paltrinieri, 2013).

De la maternelle au lycée, les lieux sont de plus en plus nombreux et se diversifient à côté de la traditionnelle salle de classe (Derouet-Besson, 2005). L'espace scolaire est non seulement un espace fortement spécifié (immédiatement reconnaissable) mais également un espace fragmenté en une diversité de lieux et d'usages (Sgard \& Hoyaux, 2006). Il nous apparaît donc important de considérer cette diversité d'espaces et de lieux lorsqu'on s'intéresse au bien-être des élèves, notamment dans l'enseignement secondaire. Certains travaux français se sont récemment intéressés à d'autres lieux d'apprentissage tels que le CDI (Durpaire \& Durpaire, 2017), ou encore la salle de permanence (Cnesco, 2017) mais sans lien direct avec la question du bien-être des élèves. Quoi qu'il en soit, les espaces de transition (escaliers, couloirs, hall d'entrée), les espaces d'intimité (vestiaires d'EPS, toilettes, infirmerie), les espaces de socialisation (cour de récréation, foyer, « cantine »), les espaces institutionnels (administration, bureau de la vie scolaire) ou encore les espaces périphériques (parvis, arrêt de bus du collège) sont quasiment absents de la réflexion. Notons toutefois une poignée de recherches qui ont croisé des lieux scolaires avec certaines composantes du bien-être. Par exemple, l'étude de Joing et Vors (2015) s'intéresse spécifiquement à la question des victimations et du climat scolaire au collège dans les vestiaires d'EPS, l'enquête de Hébert et Dugas (2017) qui interroge le sentiment de sécurité dans différents lieux de l'espace scolaire au collège ou encore les travaux de Mazalto (2017a ; 2017b) qui questionne le lien entre l'agencement des espaces scolaires, le bien-être et la réussite des élèves.

Comme le soulignent Florin et Guimard (2017 : 48), le fait «d'être globalement satisfait de l'école n'implique pas nécessairement une évaluation positive de toutes les expériences vécues dans ce contexte ». Aussi, nous soutenons l'idée que le fait de se sentir bien dans son établissement ou en salle de classe n'implique pas nécessairement un niveau de bien-être subjectif élevé dans chacun des lieux d'un établissement scolaire.

Il semble donc nécessaire d'accorder de l'importance à chacun de ces lieux lorsqu'on s'intéresse au bien-être des élèves, et de considérer que le bien-être affectif de l'élève puisse varier en fonction des lieux fréquentés. Ensuite, il apparaît essentiel d'étudier la dynamique de ce bien-être et d'examiner ce qui explique le niveau de bien-être d'un élève dans un lieu de l'espace scolaire.

\footnotetext{
${ }^{1}$ Parfois renommés « CCC » (Centre de Connaissances et de Culture)
} 
Variation du bien-être des élèves en fonction des lieux fréquentés :

le rôle du climat scolaire, de la perception du lieu

et de la satisfaction des besoins

La qualité des expériences vécues dans un lieu peut être influencée par une diversité de facteurs. Dans cette recherche, nous avons choisi d'en examiner trois : le climat scolaire (à travers notamment le sentiment de sécurité et la fréquence des victimations), la perception de l'environnement physique et le degré de satisfaction des besoins des élèves.

Bien-être et climat scolaire

Certains facteurs de climat scolaire sont intimement liés au bien-être des élèves. C'est le cas du sentiment de sécurité qui constitue un facteur de protection important pour le bien-être des élèves (Samdal et al., 1999; Lester et Cross., 2015), de la fréquence des victimations par les pairs qui impacterait négativement la satisfaction scolaire (Ladd et al., 1996; Verkuyten \& Thijs, 2002; Wachs, 2012) ou encore du climat de classe à travers notamment un climat d'entraide et de soutien (Baker et al., 2003).

De nombreux travaux s'accordent également sur l'importance de la qualité de la relation enseignant-élève sur le niveau de bien-être subjectif de l'élève (Tian et al., 2016) et leur satisfaction scolaire quel que soit le contexte culturel (Tomyn \& Cummins, 2011 ; Baker, 1999 ; Danielsen et al., 2009 ; Epstein \& McPartland, 1976 ; Hui \& Sun, 2010 ; Kong, 2008 ; Samdal et al., 1999 ; Verkuyten \& Thijs, 2002 ; Florin \& Guimard, 2017 pour une revue de littérature sur le sujet).

Si le lien entre climat et bien-être est souvent envisagé, pour autant aucun travail empirique, à notre connaissance, ne s'interroge sur le lien dynamique entre le bien-être de l'élève et la variation du climat (sentiment de sécurité, victimation...) en fonction du lieu fréquenté. La plupart des études ne distingue pas les lieux : soit elles s'appuient sur une mesure générale du bien-être scolaire à l'échelle de l'établissement, soit elles s'ancrent dans le contexte spécifique de la classe.

Bien-être et environnement physique

Certaines études postulent que l'environnement éducatif (au niveau architectural ou organisationnel par exemple) impacte le bien-être des élèves et constitue une explication possible à la baisse des scores associés à l'entrée au collège (Eccles et al., 1993). En accord avec la théorie de «person-environment fit $»^{2}$ (voir Hunt, 1975), Eccles et Migley (1989) proposaient déjà que le déclin motivationnel et comportemental pouvait résulter d'un environnement éducatif inapproprié pour les jeunes collégiens ${ }^{3}$. Ainsi, le bien-être (au sens large) serait influencé par un ajustement entre des caractéristiques individuelles et des caractéristiques de l'environnement (ici l'école). Notre travail s'inscrit dans cette lignée de pensée en explorant plus particulièrement le niveau 5 du modèle d'Eccles et Roeser (2011) (qui décrit sept niveaux d'influence de l'environnement), à savoir celui des structures organisationnelle et fonctionnelle (incluant les ressources matérielles) de l'établissement qui peuvent jouer un rôle dans le bien-être des jeunes adolescents.

Par ailleurs Barett et ses collaborateurs (2015) se sont récemment intéressés à l'impact des caractéristiques physiques d'un lieu sur le progrès des élèves (et non le bien-être). Les résultats de leur étude montrent que les progrès des élèves sont impactés par les caractéristiques physiques des écoles primaires et notam-

\footnotetext{
${ }^{2}$ Ajustement personne-environnement

3 «Early adolescents in junior high schools » dans leur étude.
} 
ment par des facteurs liés au confort ${ }^{4}$ (comme la luminosité des salles de classe ou encore la qualité de l'air), des facteurs d'appartenance ${ }^{5}$ (salles personnalisées par exemple) et par le niveau de stimulation (comme la couleur des murs) ${ }^{6}$. D'autres études menées dans des contextes culturels et sociaux différents ont également conclu à l'existence d'un lien entre les caractéristiques physiques de l'environnement scolaire et les comportements sociaux, cognitifs et/ou affectifs des élèves (Abbas \& Othman, 2010 ; Maxwell \& Chmielewski, 2008, Martensson et al., 2009), de même qu'un lien entre l'environnement physique et les performances scolaires des élèves (Bako-Biro et al., 2012 ; Winterbottom \& Wilkins, 2009).

Ces travaux montrent l'importance de s'intéresser aux caractéristiques physiques d'un lieu et à la perception qu'en ont les élèves lorsqu'on questionne le bien-être à l'école. Pourtant, nous n'avons recensé que très peu d'études empiriques s'intéressant à l'analyse de l'espace scolaire et à son effet sur le bien-être des élèves dans le contexte français. D'une manière générale, il semble qu'en France la manière de penser l'espace et l'architecture scolaire soit assez éloignée des préoccupations de climat et de bien-être des élèves (Hébert \& Dugas, 2015).

Bien-être et satisfaction des besoins

Même si certaines études montrent la stabilité du bien-être (Cummins, 2010), la plupart d'entre elles rappellent que le bien-être de chacun est susceptible de varier d'un jour à l'autre et d'un environnement à l'autre (Reis et al., 2000). Une grande partie de cette fluctuation intra-individuelle peut se comprendre en examinant comment l'environnement quotidien (qui change constamment) permet de satisfaire les besoins de l'individu et notamment les besoins psychologiques fondamentaux (Reis, 1994 ; Ryan, 1995 ; Sheldon \& Kasser, 1995 ; Reis et al., 2000). De nombreuses études se sont attachées à étudier le lien entre le bien-être subjectif et la satisfaction des besoins psychologiques fondamentaux dans différents domaines (e.g., Sheldon et al., 1995, 1996, 1997 ; Reis et al., 2000 dans un contexte général ; Gillet et al., 2015 ; Huyghebaert et al., 2017 ; Baard et al., 2004 dans le contexte professionnel ; Adie et al., 2012 dans le contexte sportif). Cependant, malgré quelques études récentes (Véronneau et al., 2005 ; Tian et al., 2015 ; Tian et al., 2016), il semble qu'une attention limitée soit accordée à l'impact de la satisfaction des besoins fondamentaux sur le bien-être subjectif des élèves dans le contexte scolaire (Tian et al., 2016).

L'hypothèse qui sous-tend l'ensemble de ces travaux est dérivée de la théorie de l'auto-détermination (Deci \& Ryan, 1991 ; 2008 ; Ryan, 1995) et postule que le bien-être est lié à la satisfaction des trois besoins psychologiques fondamentaux : l'autonomie, l'affiliation et la compétence. La théorie des besoins psychologiques fondamentaux («The Basic Psychological Needs Theory », Deci \& Ryan 1985, 1991, 2000 ; Ryan \& Deci 2000, 2002) postule en effet que les facteurs environnementaux (comme le soutien social par exemple, mais aussi pour notre étude comme la fonctionnalité, l'agencement et la perception d'un lieu) peuvent affecter le bien-être subjectif en agissant sur la satisfaction des besoins (rôle médiateur). Ainsi, selon Deci et Ryan (1991, 2008), les situations qui favorisent l'autonomie, l'appartenance sociale (ou affiliation) et la compétence devraient

\footnotetext{
${ }^{4}$ Facteur nommé « Naturalness » selon le modèle des auteurs

${ }^{5}$ Facteur nommé « Individualisation » selon le modèle des auteurs.

${ }^{6}$ Le modèle conceptuel présenté par les auteurs est défini par le sigle SIN : « Stimulation Individualisation Naturalness », qui caractérise les trois dimensions physiques permettant de mieux comprendre l'expérience humaine dans les espaces construits.
} 
améliorer le bien-être alors que les facteurs qui nuisent à ces besoins auraient tendance à le nuire (Bartholomew et al., 2011).

Dans le contexte scolaire, Baker et ses collaborateurs (2003)7 affirment que l'école fonctionne comme un environnement psychologiquement sain lorsqu'elle répond efficacement aux besoins de développement des adolescents. Par ailleurs Laguardia et Ryan $(2000,296)$ indiquent qu'une «classe qui encourage l'exercice de l'autonomie, le sentiment de compétences et d'affiliation interpersonnelle est une classe qui engage davantage l'élève, favorise son succès, et [...] augmente son bien-être psychologique ».

Cependant, lorsque des études empiriques sont menées en contexte scolaire, le bien-être et la satisfaction des besoins sont envisagés, une fois de plus, de manière globale (c'est-à-dire à l'échelle de l'établissement sans distinction des lieux, ou à l'échelle de la classe uniquement). En témoigne l'outil de Tian et ses collaborateurs (2014) permettant de mesurer la satisfaction et la frustration des besoins psychologiques fondamentaux en contexte scolaire qui fait exclusivement référence à l'établissement («at school») et à la relation enseignant-élève dans chacun des items ( «I can decide for myself how to do things at school »; « Teachers and classmates care about me at school » par exemple).

À ces besoins psychologiques, il nous semble essentiel d'ajouter la satisfaction des besoins physiologiques, notamment celui de repos, compte tenu du fait que l'adolescence est une période sensible marquée par des transformations physiologiques nombreuses et un manque de sommeil souvent important (Colrain et Baker, 2011 ; Feinbeig et Campbell, 2010).

\section{MÉTHODE}

\section{Participants}

Un courrier électronique de sollicitation a été adressé à 11 collèges des Hauts de France dans lesquels nous avions un correspondant ${ }^{8}$. Sur les 11 chefs d'établissements contactés, 10 ont répondu favorablement. Deux mille vingt-huit élèves issus de ces 10 établissements ont alors participé à l'étude. L'échantillon ne comporte pas d'élèves issus d'une Section d'Enseignement Général et Professionnel Adapté (SEGPA). La répartition selon le niveau de classe et le sexe des élèves est présentée dans le Tableau 1.

\begin{tabular}{cccc} 
Participants & Total & Filles & Garçons \\
\hline$N$ & 2028 & 970 & 1041 \\
\hline 6ème & 612 & 301 & 311 \\
5ème & 583 & 281 & 299 \\
4ème & 390 & 169 & 215 \\
3ème & 440 & 219 & 216 \\
Non renseigné & 3 & - & - \\
\hline
\end{tabular}

Tableau 1 : Niveau de classe et sexe des participants

\footnotetext{
${ }^{7}$ « Developmental-ecological framework».

${ }^{8}$ Il s'agissait d'un enseignant titulaire avec qui nous avons l'habitude de travailler.
} 
Nous avons également questionné le sentiment de compétence scolaire perçu des élèves grâce à un item unique «Je suis un(e) bon (ne) élève » pour lequel les élèves devaient se positionner sur une échelle de type Lickert en 5 points (de 1 : pas du tout d'accord ; à 5 : totalement d'accord). L'échantillon était composé de 177 élèves ne se percevant pas comme bon-ne élève ${ }^{9}(9 \%), 1172$ élèves étant d'accord avec le fait d'être un-e bon-ne élève ${ }^{10}(58 \%)$ et 673 élèves ayant un avis mitigé concernant le fait d'être un-e bon-ne élève ${ }^{11}(33 \%)^{12}$.

Sur les 10 établissements, l'échantillon compte huit collèges urbains, un collège rural et un établissement semi-rural. Les effectifs d'élèves sont variables : trois collèges comptent moins de 400 élèves, quatre établissements accueillent entre 400 et 600 élèves et trois autres en accueillent plus de 600.

\section{Instrument d'enquête}

Cette étude repose sur un questionnaire construit par notre équipe permettant d'évaluer le sentiment de sécurité, l'ordre scolaire, la victimation, la perception du lieu, la satisfaction des besoins d'autonomie, d'affiliation et de repos/détente ainsi que la qualité des expériences vécues (bien-être affectif) par les élèves dans chacun des 18 lieux retenus pour l'étude. Afin de limiter la longueur du questionnaire et le biais de lassitude chez les élèves, cinq versions du questionnaire (ce sont les mêmes questions dans chaque version mais chaque version questionne des lieux différents) sont proposées aux élèves. La version 1 du questionnaire se consacre aux salles de classe, au bâtiment administratif et au garage à vélos; la version 2 s'intéresse au CDI, au hall d'entrée, au bureau du $\mathrm{CPE}^{13}$ et à l'arrêt de bus du collège ; la version 3 questionne la salle de permanence (ou salle d'études), la cour de récréation, le parvis (communément appelé la "grille » par les élèves) et le restaurant scolaire (la « cantine ») ; la version 4 concerne la salle de sport, les toilettes et les escaliers; enfin la version 5 examine les couloirs, les vestiaires d'EPS, le bureau de la vie scolaire et les casiers. L'élève est invité à ne pas répondre aux questions s'il ne fréquente pas ou peu le lieu (moins de deux fois par an).

\begin{tabular}{lccc}
\multicolumn{1}{c}{ Participants } & Total & Filles & Garçons \\
\hline \multicolumn{1}{c}{$\mathrm{N}$} & 2028 & 970 & 1041 \\
\hline Version 1 : Salle de classe, administration, garage à vélos & 396 & 195 & 198 \\
Version 2 : CDI, hall d'entrée, bureau du CPE, arrêt de bus du & 429 & 211 & 214 \\
Version 3 : permanence, cour de récréation, parvis, cantine & 417 & 212 & 203 \\
Version 4 : salle de sport, toilettes, escaliers & 412 & 183 & 223 \\
Version 5 : Couloirs, Vestiaires EPS, bureau vie scolaire, casiers & 368 & 169 & 197 \\
\hline
\end{tabular}

Tableau 2 : Nombre de répondants par version du questionnaire

Les cinq versions du questionnaire ont été distribuées aléatoirement aux élèves, le nombre de répondants pour chaque lieu est donc d'environ $20 \%$ de l'échantillon total. La répartition du nombre de répondants par version du ques-

\footnotetext{
${ }^{9}$ Réponse 1 ou 2 sur l'échelle en 5 points.

${ }^{10}$ Réponses 4 ou 5 sur l'échelle en 5 points.

${ }^{11}$ Réponse 3 sur l'échelle en 5 points.

126 élèves n'ont pas renseigné cet item.

${ }^{13}$ Conseiller Principal d'Éducation.
} 
tionnaire est présentée Tableau 2. Les caractéristiques socio-démographiques (sexe, niveau de classe, type d'établissement) de l'échantillon sont indépendantes de la version du questionnaire reçue.

Mesure du bien-être affectif des élèves

La qualité des expériences vécues par les élèves (bien-être affectif) dans chacun des lieux étudiés a été examinée grâce à la version française ${ }^{14}$ de l'échelle de Diener et de ses collaborateurs (2009) : the Scale of Positive and Negative Experience (SPANE), version validée par Martin-Krumm et ses collaborateurs en 2017. L'outil demande au répondant de réfléchir à ce qu'il a fait au cours des quatre dernières semaines (dans le lieu spécifié) et d'indiquer à quel point, sur une échelle de fréquence en cinq points (de 1 : très rarement ou pratiquement jamais, à 5 : très souvent ou toujours) il a ressenti les sensations qui figurent dans la liste. Cette dernière comporte douze items reprenant six sensations positives (exemple : «je me suis senti(e) bien ») et six sensations négatives (exemple : «je me suis senti(e) triste).

L'indice de bien-être affectif a été calculé conformément aux indications des auteurs. Il peut prendre toutes les valeurs entre -24 et +24 . Un score proche de 24 , signifie que l'élève ne vit que des sensations et expériences positives dans ce lieu. Un score négatif signifie que les expériences vécues dans le lieu sont régulièrement associées à des sensations négatives et témoignent donc d'un niveau de bien-être subjectif faible.

La perception du lieu physique

Les élèves devaient évaluer sur une échelle de type Lickert en 5 points (de 1 : pas du tout d'accord, à $5:$ totalement d'accord) la perception physique du lieu à travers six qualificatifs (trois positifs : «c'est un lieu agréable », «... accueillant », «... propre »; et trois négatifs : «c'est un lieu triste », «... repoussant », «... sale »). L'indice final de «perception du lieu» est obtenu en moyennant les scores des six items après avoir inversé ceux des items négatifs.

Le sentiment de sécurité

Les élèves devaient évaluer sur une échelle de type Lickert en 5 points (de 1 : pas du tout d'accord, à 5 : totalement d'accord) s'ils se sentaient en sécurité («je me sens en sécurité ») dans le lieu en question.

La victimation

Les élèves devaient évaluer sur une échelle de fréquence en quatre points (de 1 : jamais ; à 4 : très souvent) s'ils étaient victimes des atteintes suivantes : ostracisme («je suis mis-e à l'écart»), atteintes verbales (« on m'insulte (ou on se moque de moi)»), atteintes physiques («on me frappe »), vols et dégradations d'affaires personnelles (« on me vole (ou on m'abîme) des affaires), atteintes à caractère sexuel («on me touche de force (caresse, baiser, on me déshabille...)»), cyber-atteintes («on me prend en photo ou on me filme sans mon autorisation »), atteintes d'incitation (« on m'oblige à faire quelque chose que je ne veux pas faire »). L'indice de victimation de l'élève est obtenu en moyennant les scores des 7 items. Plus l'indice se rapproche de quatre, plus l'élève est victime d'atteintes régulières de toutes formes (harcèlement scolaire sévère); plus il se rapproche de 1 , moins l'élève est victime d'atteintes quelle que soit la nature.

\footnotetext{
${ }^{14}$ Version en ligne :

http://internal.psychology.illinois.edu/ ediener/Documents/SPANE_French.pdf
} 
L'ordre scolaire

Les élèves devaient évaluer sur une échelle de fréquence en quatre points (de $1:$ jamais ; à 4 : très souvent) dans quelle mesure les dérégulations scolaires se manifestaient dans le lieu en question. Les dérégulations scolaires questionnées sont les suivantes: les bagarres, les jeux dangereux, le fait de faire des choses interdites par le règlement. L'indice « ordre scolaire » du lieu est obtenu en moyennant les scores des trois items. Plus l'indice se rapproche de quatre, plus le climat est propice au désordre scolaire ; à l'inverse, plus le score se rapproche de un, plus le climat respecte les règles institutionnelles établies.

La satisfaction du besoin d'affiliation

Le besoin d'affiliation renvoie au fait d'entretenir de bonnes relations interpersonnelles et de se lier positivement à des personnes qui sont importantes pour soi. Dans ce travail, nous avons cherché à évaluer la satisfaction de ce besoin au travers de deux items significatifs et particulièrement prédicteurs (Reis et al., 2000) : « je discute de choses intéressantes » et «je me sens compris-e et apprécié-e par les autres». Pour chacun de ces items, l'élève devait évaluer sur une échelle de type Lickert en cinq points (de $1:$ pas du tout d'accord; à 5 : totalement d'accord) dans quelle mesure il était d'accord avec les assertions. L'indice de satisfaction du besoin d'affiliation est obtenu en moyennant les scores des deux items. Plus l'indice se rapproche de quatre, plus les activités de l'élève dans le lieu en question permettent de satisfaire son besoin d'affiliation.

La satisfaction du besoin d'autonomie

Le besoin d'autonomie est comblé lorsque les activités sont librement consenties et en accord avec l'individu. Dans ce travail, nous avons cherché à évaluer la satisfaction de ce besoin au travers de deux items $: \ll$ je fais des activités qui me plaisent » et « je peux réaliser des choses par moi-même (je peux entreprendre)». Pour chacun de ces items, l'élève devait évaluer sur une échelle de type Lickert en cinq points (de $1:$ pas du tout d'accord; à 5 : totalement d'accord) dans quelle mesure il était d'accord avec les assertions. L'indice de satisfaction du besoin d'autonomie est obtenu en moyennant les scores des deux items. Plus l'indice se rapproche de 4, plus les activités de l'élève dans le lieu en question sont librement consenties.

Notons que nous n'avons pas jugé pertinent dans cette étude d'évaluer la satisfaction du besoin de compétence ${ }^{15}$. En effet, la question de la satisfaction du besoin de compétence dans les escaliers, la cour de récréation ou encore les toilettes par exemple ne nous a pas semblé adéquate dans ce travail.

La satisfaction du besoin physiologique

de repos et de détente

Dans ce travail, nous avons cherché à évaluer la satisfaction du besoin physiologique de repos et de détente de l'adolescent au travers de deux items : « je me repose » et « je me détends ». Pour chacun de ces items, l'élève devait évaluer sur une échelle de type Lickert en cinq points (de $1:$ pas du tout d'accord ; à 5 : totalement d'accord) dans quelle mesure il était d'accord avec les assertions. L'indice de repos/détente est obtenu en moyennant les scores des deux items. Plus l'indice

\footnotetext{
${ }^{15}$ Le besoin de compétence renvoie au besoin de se sentir efficace et performant, et à la capacité de produire les effets et les résultats désirés. Il est l'un des trois besoins psychologiques fondamentaux (Deci \& Ryan 1985, 1991, 2000 ; Ryan \& Deci 2000, 2002).
} 
se rapproche de quatre, plus les activités de l'élève dans le lieu en question permettent de satisfaire ce besoin.

\section{Analyses statistiques}

Identification des lieux dans lesquels le niveau de bien-être est significativement élevé ou faible

Des méthodes statistiques standard ont été utilisées pour calculer les moyennes et les écarts-types des différents scores. Afin de détecter les effets significatifs des différents lieux sur les scores de bien être affectif, le test de Student pour un échantillon est utilisé en comparant les valeurs de chaque lieu à la valeur moyenne de l'ensemble des lieux. La correction de Bonferronni est utilisée pour fixer le seuil de significativité à 0,05 .

Identification des facteurs explicatifs du bien-être

dans les différents lieux de l'espace scolaire

Une analyse en régression multiple a été effectuée pour identifier les facteurs les plus explicatifs de l'indice de bien-être affectif dans un lieu. Le score de bien être affectif a été défini comme variable dépendante ; les scores de «besoin d'affiliation », « besoin d'autonomie », besoins physiologiques », « perception du lieu », « sentiment de sécurité », « victimation » et « dérégulations scolaires » ont été définis comme variables prédictives.

L'ensemble des procédures statistiques a été réalisé par le logiciel Statistica 10.0. Le seuil de significativité a été fixé à $\mathrm{p}=0.05$.

Procédures

L'enquête de terrain a été menée entre mars et avril 2017 dans les 10 établissements des Hauts-de France préalablement contactés. Un ou plusieurs enseignants référents de l'établissement ont été formés pour organiser la passation du questionnaire ${ }^{16}$. Ils devaient respecter scrupuleusement des consignes de passation formalisées à l'écrit. Celles-ci reprenaient le discours de passation à tenir aux élèves (introduction de l'étude, rappel de l'anonymat, précisions à apporter concernant l'échelle de réponse pour les élèves de 6ème notamment) et rappelaient l'importance d'une distribution aléatoire.

Conformément à la loi informatique et au règlement européen sur la protection des données, une déclaration a été faite et une autorisation a été enregistrée et publiée sous le numéro 2018-7 au registre des traitements de l'Université de Lille.

\section{RÉSULTATS}

L'objectif de cette étude est d'examiner la dynamique du bien-être au collège en repérant les lieux où les élèves vivent le plus d'expériences positives ainsi que d'identifier les facteurs explicatifs de cette dynamique.

\footnotetext{
${ }^{16}$ Le questionnaire a fait préalablement l'objet d'un pré-test auprès d'une classe de $6^{\mathrm{e}}$ et d'une classe de $3^{\mathrm{e}}$.
} 
Bien-être au collège

en fonction des lieux fréquentés

Tous lieux confondus, l'indice global moyen de bien-être affectif est de 8.94 avec des indices individuels allant de -24 à +24 (7709 observations au total $)^{17}$.

Les indices de bien-être affectif dans les différents lieux du collège sont présentés dans le Tableau 3. Les résultats issus d'une analyse comparative montrent que le bien-être affectif est significativement élevé au CDI (I = 11.31; ET $=9.55 ; \mathrm{p}<.0027)^{18}$, en salle de sport $(\mathrm{I}=11.77 ; \mathrm{ET}=8.70 ; \mathrm{p}<.0027)$, à la « cantine » $(\mathrm{I}=13.76 ; \mathrm{ET}=8.47 ; \mathrm{p}<.0027)$, ainsi que dans la cour de récréation $(\mathrm{I}=13.86 ; \mathrm{ET}=8.27 ; \mathrm{p}<.0027)$. Notons qu'au seuil $\mathrm{p}<.5$, l'indice de bien-être affectif est également significativement élevé dans les vestiaires d'Éducation Physique et Sportive $(\mathrm{I}=9.95 ; \mathrm{ET}=8.37 ; \mathrm{p}<.05)$.

À l'inverse, les résultats indiquent un indice de bien-être significativement bas dans les lieux suivants : le bureau du $\mathrm{CPE}^{19}(\mathrm{I}=2.66 ; \mathrm{ET}=10.62 ; \mathrm{p}<.0027)$, les toilettes $(\mathrm{I}=2.83 ; \mathrm{ET}=9.39 ; \mathrm{p}<.0027)$, le bureau de la vie scolaire (I $=6.51 ; \mathrm{ET}=9.56 ; \mathrm{p}<.0027)$ et le bâtiment administratif $(\mathrm{I}=6.41 ; \mathrm{ET}=9.48 ; \mathrm{p}$ $<.0027)$. Notons qu'au seuil $\mathrm{p}<.05$, l'indice de bien-être des élèves est également significativement faible dans les escaliers $(\mathrm{I}=7.57 ; \mathrm{ET}=8.76 ; \mathrm{p}<.05)$.

\begin{tabular}{|c|c|c|c|c|c|c|c|}
\hline Variable & Moyenne & $\begin{array}{l}\text { Ec- } \\
\text { Type }\end{array}$ & $N$ & $\begin{array}{c}\text { Erreur- } \\
\quad T\end{array}$ & $\begin{array}{c}\text { Valeur } \\
\text { de réfé- } \\
\text { rence }\end{array}$ & Valeur $t$ & $p$ \\
\hline $\begin{array}{l}\text { Salles de } \\
\text { classe }\end{array}$ & 8.512 & 8.975 & 386 & 0.456 & 8.94 & -0.936 & 0.349638 \\
\hline CDI & $11.310^{* *}$ & 9.550 & 360 & 0.503 & 8.94 & 4.708 & $0.000004 * *$ \\
\hline Permanence & 8.264 & 9.326 & 410 & 0.460 & 8.94 & -1.466 & 0.143400 \\
\hline Salle de sport & $11.772 * *$ & 8.707 & 397 & 0.437 & 8.94 & 6.481 & $0.000000 * *$ \\
\hline Cour de récré & 13.863 & 8.279 & 394 & 0.417 & 8.94 & 11.802 & $0.000000 * *$ \\
\hline «Cantine » & $13.761 * *$ & 8.479 & 378 & 0.436 & 8.94 & 11.054 & $0.000000 * *$ \\
\hline Toilettes & $2.831 * *$ & 9.394 & 373 & 0.486 & 8.94 & -12.557 & $0.000000 * *$ \\
\hline Vestiaires EPS & $9.958 *$ & 8.377 & 348 & 0.449 & 8.94 & 2.268 & $0.023917 *$ \\
\hline Escaliers & $7.570^{*}$ & 8.764 & 338 & 0.476 & 8.94 & -2.872 & $0.004326^{*}$ \\
\hline Couloirs & 8.945 & 7.503 & 350 & 0.401 & 8.94 & 0.013 & 0.989549 \\
\hline Casiers & 8.630 & 8.179 & 219 & 0.552 & 8.94 & -0.56 & 0.575577 \\
\hline Hall d'entrée & 8.882 & 8.633 & 358 & 0.456 & 8.94 & -0.126 & 0.899567 \\
\hline Parvis & 9.401 & 9.883 & 379 & 0.507 & 8.94 & 0.909 & 0.363624 \\
\hline Arrêt de bus & 8.145 & 9.663 & 176 & 0.728 & 8.94 & -1.09 & 0.276910 \\
\hline Garage à vélos & 8.748 & 7.601 & 140 & 0.642 & 8.94 & -0.298 & 0.766162 \\
\hline Administration & $6.417 * *$ & 9.484 & 255 & 0.593 & 8.94 & -4.246 & $0.000030 * *$ \\
\hline Bureau CPE & $2.668 * *$ & 10.627 & 220 & 0.716 & 8.94 & -8.752 & $0.000000 * *$ \\
\hline Vie scolaire & $6.510 * *$ & 9.567 & 312 & 0.541 & 8.94 & -4.485 & $0.000010 * *$ \\
\hline
\end{tabular}

Tableau 3 : Indice de bien-être affectif en fonction des lieux fréquentés

\footnotetext{
${ }^{17} 2028$ élèves ayant renseigné 3 à 4 lieux différents (en fonction de la version du questionnaire).

${ }^{18}$ Nous appliquons la correction de Bonferroni au seuil initial de $\mathrm{p}=0.05$ : étant donné qu'il y a 18 lieux, la valeur corrigée de $\mathrm{p}=0.05 / 18=0.0027$

${ }^{19}$ Conseiller Principal d'Éducation
} 
Effet du sexe sur le bien-être affectif

dans chacun des lieux de l'espace scolaire

Les résultats ne révèlent aucun effet du sexe sur la qualité des expériences vécues (bien-être affectif), quel que soit le lieu étudié.

Effet du niveau de classe sur le bien-être affectif

dans chacun des lieux de l'espace scolaire

Tous lieux confondus, les résultats révèlent que les élèves de 6ème vivent davantage d'expériences positives comparativement aux élèves de 3ème pour qui l'indice moyen de bien-être affectif est, lui, significativement bas ( $\mathrm{I}=10.6$ pour les élèves de 6ème vs $\mathrm{I}=7.8$ pour les 4ème et $\mathrm{I}=7.7$ pour les 3ème, $\mathrm{p}<.05$ ).

Plus spécifiquement, les élèves de 6ème obtiennent un indice moyen de bien-être affectif significativement plus élevé que le reste de l'échantillon pour le hall d'entrée ( 11.66 vs $8.88 ; \mathrm{p}<.05)$, la salle de permanence/études $(10,26$ vs $8,26 ; \mathrm{p}<.05)$, les toilettes $(5.9$ vs $2.83 ; \mathrm{p}<.05)$, le bureau de la vie scolaire $(9.4$ vs $6.51 ; \mathrm{p}<.05)$, les salles de classe $(10,47$ vs $8.51 ; \mathrm{p}<.05)$ et la salle de sport $(13,65$ vs $11.77 ; \mathrm{p}<.05)$.

A l'inverse les élèves de 3ème vivent davantage d'expériences négatives comparativement aux autres élèves dans les lieux suivants : les casiers $(6,15$ vs $8.63 ; \mathrm{p}<.05)$, les couloirs (6.71 vs $8.94 ; \mathrm{p}<.05)$, les salles de classe (4.67 vs $8.51 ; \mathrm{p}<.05)$ et la salle de sport $(9,57$ vs $11.77 ; \mathrm{p}<.05)$.

Effet du sentiment de compétence scolaire perçu

sur le bien-être affectif dans chacun des lieux de l'espace scolaire

Tous lieux confondus, les élèves ayant une perception de compétence scolaire faible vivent davantage d'expériences négatives $(7.37$ vs $8.94, \mathrm{p}<.05)$. Il en est de même pour les élèves ayant un jugement moyen de leur compétence scolaire (7.89 vs $8.94, p<.05)$. À l'inverse, les élèves se percevant bon-ne élève obtiennent un indice moyen de bien-être affectif significativement plus élevé que le reste de l'échantillon $(9.8$ vs $8.94, \mathrm{p}<.05)$.

Plus spécifiquement, les élèves ayant une perception de compétence scolaire faible déclarent vivre davantage d'expériences négatives comparativement aux autres élèves lorsqu'ils fréquentent les casiers ( 4.12 vs $8.63, p<.05)$, les salles de classe $(6.47$ vs $8.51, p<.05)$ ou la salle de sport $(6.38$ vs $11.77, \mathrm{p}<.05)$.

À l'inverse, les élèves en accord avec le fait d'être un-e bon-ne élève déclarent vivre davantage d'expériences positives dans les salles de classe ( $\mathrm{I}=10.07 \mathrm{vs}$ $\mathrm{I}=8.51, \mathrm{p}<.05)$.

Rôle des variables à l'étude sur le bien-être affectif

dans un lieu de l'espace scolaire, tous lieux confondus

Cinq mille quatre-vingt-onze $(5091)^{20}$ observations ont été retenues pour tester le modèle de régression multiple examinant la valeur prédictive des variables liées au climat, à la perception du lieu et à la satisfaction des besoins des élèves pour le bien-être affectif dans un lieu. Les résultats montrent qu'une partie importante de l'indice de bien-être affectif $(\mathrm{R} 2=0,51)$ est expliquée par les cinq variables suivantes : la perception du lieu, l'affiliation, l'autonomie, la victimisation et le sentiment de sécurité. Ainsi, $51 \%$ de la variance du bien-être affectif est expliqué par les cinq variables.

${ }^{20} 2028$ élèves ayant répondu pour trois ou quatre lieux. 7709 observations au total dont 2618 qui n’ont pas été prises en compte (non réponse à au moins un des critères). 
Dans ce modèle, une perception positive du lieu $(\mathrm{B}=0.40 ; \mathrm{p}<.05)$ associée à des activités permettant de satisfaire le besoin d'affiliation $(B=0,17$; p $<.05)$ et d'autonomie des élèves $(\mathrm{B}=0,17, \mathrm{p}<.05)$ ainsi qu'à un faible niveau de victimisation $(\mathrm{B}=-0,16 ; \mathrm{p}<.05)$ et un sentiment de sécurité élevé $(\mathrm{B}=0.07, \mathrm{p}$ $<.05)$ constituent des facteurs favorisant un niveau de bien-être affectif élevé dans un lieu.

L'analyse en régression multiple met donc en évidence que toutes les variables, exceptée celle de l'ordre scolaire, peuvent être considérées comme des variables prédictives. Cependant, la valeur du coefficient Bêta le plus élevé concerne la perception du lieu ; viennent ensuite dans une proportion équivalente la satisfaction du besoin d'affiliation et d'autonomie. Enfin, la variable «victimisation » dont le coefficient Bêta est négatif participe également pour une part significative à l'explication de la variance du score bien-être.

\begin{tabular}{lccc} 
& Béta & $\begin{array}{c}\text { Erreur } \\
\text { Type }\end{array}$ & $p$ \\
\hline Perception du lieu & 0.40 & 0.01 & $0.00^{*}$ \\
Affiliation & 0.17 & 0.01 & $0.00^{*}$ \\
Autonomie & 0.17 & 0.01 & $0.00^{*}$ \\
Victimisations & -0.16 & 0.01 & $0.00^{*}$ \\
Sentiment de sécurité & 0.0724 & 0.01 & $0.00^{*}$ \\
Besoins physiologiques & 0.0713 & 0.01 & $0.00^{*}$ \\
Dérégulations scolaires & 0.0052 & 0.01 & 0.64
\end{tabular}

Tableau 4 : Récapitulatif du modèle explicatif du bien-être affectif dans un lieu de l'espace scolaire (régression multiple; tous espaces confondus)

\section{DISCUSSION}

L'objet du présent travail visait à examiner et à expliquer l'hypothèse d'une fluctuation du bien-être subjectif (composante affective) en fonction du lieu fréquenté par l'élève au sein de l'espace scolaire.

\section{Un bien-être différencié selon le lieu}

Les résultats confirment l'hypothèse d'un bien-être différencié en fonction des lieux fréquentés. En effet, les élèves vivent davantage d'expériences positives dans la cour de récréation, la «cantine », au CDI ou encore lorsqu'ils fréquentent le gymnase d'Education Physique et Sportive (EPS). Les vestiaires d'EPS sont également un lieu apprécié des élèves. Ces lieux font partie des espaces de socialisation (Pourchet \& Cerfontaine, 2004) à l'école où les opportunités de partager un moment de détente, de discussion et/ou de travail avec les camarades sont sans doute plus fréquentes et importantes que dans d'autres lieux. Cette hypothèse explicative est en partie appuyée par les résultats de l'analyse linéaire de régression multiple qui montrent qu'une partie du bien-être des élèves est expliquée par le fait de vivre des activités permettant de satisfaire leur besoin d'affiliation.

Cela renvoie également à la volonté de socialisation qu'ont les élèves qui poursuivent deux objectifs majeurs : suivre le cours (apprendre) et se socialiser (Allen, 1986). Le fait que les élèves déclarent vivre davantage d'expériences et de sensations positives dans les vestiaires d'EPS appuie, selon nous, cette hypothèse explicative. Ce lieu pourtant habituellement qualifié de «problématique » par les adultes en raison d'une surveillance des élèves qui échappe en partie à leur vigi- 
lance (Joing \& Vors, 2014), est ici évalué positivement par les élèves en terme de bien-être affectif, sans doute en raison du fait qu'il offre aux élèves un moment de convivialité et une occasion privilégiée d'échanger et de discuter entre pairs de même sexe. Ce résultat concorde avec le travail de Joing \& Vors (2014) qui mentionnait une évaluation plus négative du climat scolaire dans les vestiaires d'EPS de la part des enseignants comparativement aux élèves. De plus, les élèves déclarent vivre des expériences également particulièrement positives lorsqu'ils fréquentent le CDI, lieu dans lequel l'apprentissage se fait souvent en autonomie par petits groupes de pairs (renforçant ainsi les besoins d'affiliation et d'autonomie).

En revanche, les résultats révèlent que le bien-être des élèves est significativement bas lorsqu'ils fréquentent la plupart des lieux dits «institutionnels » (le bureau du CPE, l'administration ou la vie scolaire), ou encore les toilettes. Le niveau de bien-être des élèves dans la plupart des lieux est probablement conditionné par les activités que les élèves y font. Nous pouvons donc aisément penser que le niveau de bien-être des élèves dans les espaces institutionnels soit lié aux raisons qui amènent les élèves à fréquenter ces lieux (pose de sanctions, la plupart du temps négatives ; entretien au sujet d'un comportement inadapté... qui amènent l'élève à fréquenter le bureau du CPE ou le bureau d'un personnel de direction). Cependant, cela interroge tout de même la qualité de la relation personnel éducatif (ou de direction) - élève. Peu d'études se consacrent à l'étude de cette relation et il semblerait intéressant de s'y attarder dans l'idée d'une amélioration globale du bien-être de l'élève.

Certaines études françaises récentes (enquête de l'AFEV en septembre 2017 ; Cnesco, 2017) ont déjà pointé du doigt la dépréciation des toilettes par les élèves ou par l'institution. Catherine Bonnety $(2014)^{21}$ évoque quelques éléments inquiétants concernant les toilettes dans les établissements scolaires. Il semble alors essentiel de s'attarder spécifiquement sur cette question afin d'identifier ce qui pourrait permettre d'améliorer la perception de ce lieu et d'augmenter ainsi le bien-être des élèves dans ce lieu. Des approches davantage qualitatives et contextualisées sont sans doute nécessaires.

L'analyse approfondie de nos données révèle quelques effets d'établissement : dans certains collèges de l'échantillon, un ou plusieurs lieux peuvent apparaître comme significativement plus problématiques (ou au contraire plus positifs) en terme de bien-être affectif comparativement aux autres établissements. Notre enquête ne permet pas d'interpréter ces données et une approche qualitative davantage contextualisée s'avère nécessaire pour identifier les raisons qui font qu'un lieu, perçu habituellement comme problématique (ou au contraire particulièrement positif) en terme de bien-être affectif des élèves peut être perçu tout autrement dans un contexte d'établissement singulier.

Le lieu scolaire doit être avant tout esthétique

et permettre de développer l'autonomie et les relations interpersonnelles

en toute sécurité

Au regard des résultats, il semble nécessaire de s'attacher non seulement à l'esthétique des lieux au sein de l'espace scolaire mais également à leur fonctionnalité (Mazalto, 2017a). Sur ce dernier point, si le lieu doit bien entendu répondre en premier à la fonction pour lequel il a été conçu, il semble également nécessaire

\footnotetext{
${ }^{21}$ Entretien avec Catherine Bonnety, article publié par François Jarraud en avril 2014 sur le site www.cafepedagogique.net.
} 
de penser les lieux de manière à promouvoir l'autonomie et les relations interpersonnelles des élèves.

En effet, les résultats montrent que le facteur le plus influent sur le niveau de bien-être des élèves dans un lieu de l'espace scolaire est l'esthétique du lieu et la perception que les élèves se font de ce lieu. Plus les élèves considèrent le lieu comme agréable, propre et accueillant, plus les expériences vécues dans ce lieu seront positives. Trop peu de travaux s'intéressent au design architectural (sensorysensitive design) en contexte scolaire avec l'idée de répondre aux attentes des principaux usagers, à savoir les élèves. L'enjeu est donc sans doute « d'aborder le bien-être dans l'architecture scolaire » (Mazalto, 2017b) en rallier les contraintes institutionnelles (obligation de surveillance des élèves par exemple) et les besoins des élèves.

Sur ce dernier point les résultats de ce travail révèlent qu'un lieu qui permet la satisfaction des besoins d'affiliation et d'autonomie des élèves a davantage de chance d'être un lieu dans lequel les élèves se sentent bien. Il semble donc nécessaire de penser la fonctionnalité des lieux autour de la satisfaction de ces besoins, tel que le préconise Mazalto (2017b) en prenant l'exemple des gradins ou en traitant la question des territoires par exemple.

Les résultats rappellent qu'un lieu ne saurait être un espace de bien-être s'il n'était pas sécuritaire pour l'élève ; d'où l'enjeu de proposer des lieux et espaces qui répondent non seulement aux attentes et besoins des élèves mais également aux attentes et contraintes institutionnelles (obligation de surveillance par exemple).

\section{Limites et perspectives}

L'approche transversale a été privilégiée pour rendre compte des fluctuations de bien-être au sein d'un établissement scolaire. Les cinq versions du questionnaire engendrent en effet autant de groupes d'élèves différents, rendant impossible l'analyse des variations intra-individuelles sur l'ensemble des 18 lieux étudiés. Si cela peut constituer une limite certaine, ce choix s'explique par la multiplicité des lieux scolaires et la nécessité de poser les mêmes questions pour chaque lieu. Si chaque élève avait répondu pour l'ensemble des 18 lieux, un biais de lassitude majeur aurait très certainement invalidé l'étude. Cette approche a néanmoins permis de montrer l'intérêt d'une approche par facettes (versus une approche globale ne différenciant pas les lieux scolaires) quand on s'intéresse au bien-être de l'élève. En effet, l'indice de bien-être affectif diffère fortement selon le lieu fréquenté. Autrement dit, le fait de se sentir bien au collège ne signifie sans doute pas que l'élève se sente automatiquement bien dans les autres lieux et inversement. Il y aurait donc bien un impact des espaces sur les usagers (Barett et al., 2015). Les investigations semblent à poursuivre dans ce sens.

Nous tenons également à souligner que, s'ils sont déjà nombreux à avoir été investigués, les lieux étudiés dans ce travail ne sont pas exhaustifs : il aurait par exemple été intéressant de s'intéresser au foyer des élèves (quand il existe), à l'infirmerie ou à d'autres lieux qui font la spécificité des établissements. De plus, nous avons fait le choix dans cette étude de considérer les salles de classe comme un lieu unique. Or, il est fort à parier qu'il y ait, du point de vue de l'élève, autant de lieux que de salles de classe et que si les élèves peuvent se sentir parfaitement bien dans une salle, ils peuvent éprouver un profond mal-être dans une autre...

Il est à noter que la période de l'année et le climat associé aux saisons (ici mars-avril) peuvent également influencer la qualité des expériences vécues dans un lieu. Une cour de récréation ne serait-elle pas davantage propice aux expé- 
riences positives lorsque le temps est ensoleillé comparativement à un contexte de froid glacial hivernal ?... Quoi qu'il en soit, il semble nécessaire de renforcer ces résultats par des approches davantage qualitatives, qui tiennent compte des spécificités des établissements scolaires.

Un enjeu important des investigations futures est de consolider l'identification des facteurs de bien-être dans un lieu. Les résultats montrent que la qualité des expériences vécues dans un lieu est conditionnée par le fait de s'y sentir en sécurité, de la percevoir positivement et de pouvoir mener des activités qui satisfassent les besoins d'autonomie et d'affiliation. Il convient de renforcer ces résultats par d'autres enquêtes de terrain pour voir comment un lieu permet de satisfaire ces conditions. Autrement dit, il nous paraît essentiel d'identifier ce qui fait qu'un lieu puisse réponde à la fois aux besoins des utilisateurs (autonomie, affiliation, sécurité) et aux contraintes et attentes institutionnelles, et permette ainsi aux élèves de se sentir bien tout en permettant aux acteurs institutionnels de répondre de leurs fonctions. Ces enquêtes davantage qualitatives permettront de différencier ce qui relève d'une perception en relation avec l'institution ou une perception concernant précisément le lieu en tant qu'espace et/ou territoire, c'est-à-dire produit d'une construction et d'une appropriation par les acteurs, d'un système socio-spatial symbolique et contextualisé (Champollion, 2008). Quoi qu'il en soit, cela nécessite sans aucun doute de se pencher vers des approches davantage qualitatives tel que le préconise Eccles et Roeser (2011 : 236) : «Parce que la plupart des recherches sont une simplification de la réalité scolaire (Lee, 2000), nous pensons que les études ethnographiques et d'observations de terrain continueront de constituer une source importante de compréhension $»^{22}$, d'autant plus que «la relation entre les bâtiments scolaires et la santé et l'apprentissage des élèves... est plus viscéralement acceptée que véritablement prouvée " ${ }^{23}$ (Baker \& Berstein, 2012, in : Barett et al., 2015 : 130).

Isabelle JOING

Univ. Lille, Univ. Artois, Univ. Littoral Côte d'Opale EA 7369 - URePSSS

Unité de Recherche Pluridisciplinaire Sport Santé Société

F-59000 Lille

Olivier VORS

Aix-Marseille Univ.

CNRS, ISM, UMR 7287

SFERE-Provence, FED 4238

Clément LLENA

Univ. Bordeaux

Laboratoire Culture, Éducation, Société, EA 743

Univ. Lille, Univ. Artois, Univ. Littoral Côte d'Opale

EA 7369 - URePSSSFrançois POTDEVIN

Univ. Lille, Univ. Artois, Univ. Littoral Côte d'Opale EA 7369 - URePSSS

\footnotetext{
${ }^{22}$ Traduction libre de «because most school research represents a "simplification" of young people's actual school experience (Lee, 2000), we believe that the need for rich observational and ethnographic studies of schooling will continue to be important sources of inquiry in the field ».

${ }^{23}$ Traduction libre de «the relationship between school buildings and student health and learning $\ldots$ is more viscerally understood than logically proven ».
} 
Abstract : This study examines the variation of pupils'experiences (the affective aspect of subjective well-being) in lower secondary school (collège) according to the places they are in and seeks to identify the factors that best predict the quality of the lived experience within a place. Much previous research has taken a global approach to well-being at school without distinguishing the different parts of the school premises. The present study postulates that this well-being varies in the course of the day according to the parts of the school the pupil is in and that this variation can be explained by the degree of satisfaction of psychological and/or physiological needs, the physical perception of the place, or the climate that prevails in it. The fieldwork was carried out on 2028 pupils of 10 collèges in the Hauts-de-France region. The results bring to light the places for which the pupils say they have the most positive experiences (playground, dining room, sports hall, library) and those for which well-being scores are significantly low (institutional parts of the school such as the school administration and education counselling offices, and the toilets). They also show that the quality of the experiences felt in a place depends on the pupil's perceiving it as pleasant, welcoming and clean, engaging in activities that contribute to the satisfaction of the needs of relatedness and autonomy, not being bullied, and feeling safe.

Keywords : spaces, junior high school, subjective well-being, relatedness, victimization, school, school climate.

\section{Bibliographie}

Abbas M. Y. \& Othman M. (2010) « Social behavior of preschool children in relation to physical spatial definition» - Procedia - Social and Behavioral Sciences 5 (935-941).

Adie J. W., Duda J. L. \& Ntoumanis N. (2012) « Perceived coach-autonomy support, basic need satisfaction and the well-and ill-being of elite youth soccer players: A longitudinal investigation» - Psychology of Sport and Exercise 13, 1 (51-59). DOI : $10.1016 / j$. psychsport.2011.07.008

Allen J. D. (1986). "Classroom Management : Students' Perspectives, Goals, and Strategies » - American Educational Research Journal 23, 3 (437459).

Baard P. P., Deci E. L. \& Ryan R. M. (2004) «Intrinsic need satisfaction : A motivational basis of performance and well-being in two work settings » - Journal of Applied Social Psychology 34, 10 (2045-2068). DOI : 10.1111/j.1559-1816.2004.tb02690.x

Baker J. A. (1999) « Teacher-student interaction in urban at-risk classrooms : Differential behavior, Relationship quality, and student satisfaction with school » - The Elementary School Journal 85 (69-80).

Baker A. J., Dilly L. J., Aupperlee J.L. \& Patil, S.A. (2003) « The Developmental Context of School Satisfaction: Schools as Psychologically Healthy Environments »-School Psychology Quarterly 18, 2 (206-221).

Baker L. \& Bernstein H. (2012) The impact of school buildings on student health and performance : a call for research. New York : McGraw-Hill Research Foundation.

Bako-Biro Zs., Clements-Croome D. J., Kochhar N., Awbi H. B. \& Williams M. J. (2012) «Ventilation rates in schools and pupils'performance » - Building and Environment 48 (215-223).

Barrett P., Davies F., Zhang Y. \& Barrett L. (2015) « The impact of classroom design on pupils' learning : Final results of a holistic, multi-level analysis » - Building and Environment 89 (118-133). 
Bartholomew K. J., Ntoumanis N., Ryan R. M. \& Thogersen-Ntoumani C. (2011) «Psychological need thwarting in the sport context: Assessing the darker sides of athletic experience »- Journal of Sport and Exercise Psychology 33 (75-102).

Champollion, P. (2008) «La territorialisation du processus d'orientation en milieux ruraux isolés et montagnards : des impacts du territoire à l'effet de territoire »-Éducation \& Formations 77 (43-53).

Cnesco (2017) Enquête sur la restauration et l'architecture scolaires. Paris.

Colrain, I.M., \& Baker, F.C. (2011) « Changes in sleep as a function of adolescent development »-Neuropsychology Review 21 (5-21).

Cummins, R. A. (2010) «Subjective Wellbeing, Homeostatically protected mood and depression : a synthesis »- Journal of Happiness Studies 11 (117). Doi.org/10.1007/s10902-009-9167-0.

Danielsen A. G., Samdal O. Hetland J. \& Wold B. (2009) « School-Related Social Support and Students' Perceived Life Satisfaction » - The Journal of Educational Research 102 (303-318).

Deci E. L. \& Ryan R. M. (1985) Intrinsic motivation and self-determination in human behavior. New York : Plenum.

Deci E. L. \& Ryan R. M. (1991) «A motivational approach to self : Integration in personality »- in : R. Dienstbier (ed.) Nebraska symposium on motivation : Perspectives on motivation 38 (237-288). Lincoln : University of Nebraska Press.

Deci E. L. \& Ryan R. M. (2000). The "what" and "why" of goal pursuits : Human needs and the selfdetermination of behavior. Psychological Inquiry, 11 (4), $227-268$. DOI : 10.1207/S15327965PLI1104_01

Deci, E. L., \& Ryan, R. M. (2008) « Hedonia, eudaimonia, and well-being : An introduction »- Journal of Happiness Studies 9 (1-11).

Derouet-Besson M. (2005) «L'apport de l'école à la construction d'une culture architecturale en France »-Revue de l'Inspection Générale 2 (419).

Diener E. (1984) «Subjective Well-Being » - Psychological Bulletin 95 (542575).

Diener E. (1994) «Assessing subjective well-being : progress and opportunities » - Social indicators research 31,2 (103-157).

Diener E., Diener C. \& Diener M. (1995) «Factors predicting the subjective wellbeing of nations » - Journal of Personality and Social Psychology 69 (851-864).

Diener E. (1996) « Traits can be powerful, but are not enough : Lessons from subjective well-being » - Journal of Research in Personality 30 (389399).

Diener E., Wirtz D., Tov W., Kim-Prieto C., Choi. D., Oishi S. \& Biswas-Diener R. (2009) «New measures of well-being: Flourishing and positive and negative feelings » - Social Indicators Research 39 (247-266).

Durpaire F. \& Durpaire J. L. (2017) «La fin d'une école, le début d'une autre ? Les évolutions architecturales des espaces de vie scolaire » - Éducation et Socialisation, Les Cahiers du CERFEE 43. Doi : 10.4000/edso.1939.

Eccles J., Midgley C. \& Adler T. (1984) «Grade-related changes in the school environment: Effects on achievement motivation»- in: J.G. 
Nicholls (ed.) The development of achievement motivation (283-331). Greenwich, CT : JAI Press.

Eccles J. S. \& Midgley C. (1989) «Stage/environment fit : Developmentally appropriate classrroms for early adolescents »- in : R. E. Ames and C. Ames (eds.) Research on motivation in education 3 (139-186). San Diego, CA : Academic Press.

Eccles J. S., Midgley C., Wigfield A., Buchanan C. M., Reuman D., Flanagan C. \& Mac Iver D. (1993) «Development during adolescence : The impact of stage-environment fit on young adolescents'experiences in schools and in families »-American Psychologist 48, 2 (90-101).

Eccles J. S. \& Roeser R. W. (2011) « School and community influences on human development »- in : M. H. Borstein and M. E. Lamb (eds.) Developmental Science : an Advanced Textbook (571-642). NY : Psychology Press.

Elmore G. M. \& Huebner E. S. (2010) «Adolescents'satisfaction with school experiences: relationships with demographics, attachment relationships, and school engagement behavior» - Psychology In The Schools 47, 6 (525-537).

Epstein J. L. \& McPartland (1976) «The Concept and Measurement of the Quality of School Life »-American Educational Research Journal 13, 1 (15-30).

Feinberg I \& Campbell I.G. (2010) «Sleep EEG changes during adolescence : an index of fundamental brain reorganization »-Brain Cognition 72 (56-65).

Ferrière S., Bacro F., Florin A. \& Guimard P. (2016) « Le bien-être en contexte scolaire : Intérêt d'une approche par triangulation méthodologique » - Cahiers Internationaux de Psychologie Sociale 111 (341-365).

Florin A. \& Guimard P. (2017) La qualité de vie à l'école. Paris : rapport commandité par le Cnesco.

Gillet N., Forest J., Benabou C. \& Bentein K. (2015) « The effects of organizational factors, psychological need satisfaction and thwarting, and affective commitment on workers'well-being and turnover intentions » - Le Travail Humain 78 (119-140). DOI : $10.3917 /$ th.782.0119

González-Carrasco M., Casas F., Malo S., Viñas F. \& Dinisman T. (2017) «Changes with age in subjective well-being through the adolescent years : Differences by gender »-Journal of Happiness studies, 18, $1(63-88)$. DOI : 10.1007/s10902-016-9717-1

Guimard P., Bacro F., Ferrière S., Florin A. \& Gaudonville T. (2017) «Évaluation du bien-être perçu des élèves : étude longitudinale à l'école élémentaire et au collège. Recherche complémentaire (BE-Scol2)»-Rapport pour la Direction de l'évaluation de la prospective et de la performance. Convention 2015 - DEPP - 028.

Hébert T. \& Dugas E. (2017) «Quels espaces scolaires pour le bien-être relationnel ? Enquête sur le ressenti des collégiens français » - Éducation et Socialisation, Les Cahiers du CERFEE 43. DOI : $10.4000 /$ edso.1904

Huebner E. S. (1994) "Preliminary development and validation of a multidimensional life satisfaction scale for children »-Psychological Assessment 6 (149-158). 
Huebner E. S. \& Gilman R. (2006) «Students who like and dislike school» Applied Research in Quality of Life 1 (139-150).

Hui E. \& Sun R. (2010) «Chinese children's perceived school satisfaction : the role of contextual and intrapersonal factors »-Educational Psychology: An International Journal of Experimental Educational Psychology 30, 2 (155-172).

Hunt D. E. (1975) «Person-Environement interaction : A challenge found wanting before it was tried»-Review of Educational Research 45 (209230).

Huyghebart T., Gillet N., Fernet C., Lahiani F. J., Chevalier S. \& Fouquereau E. (2017) « Investigating the Longitudinal Effects of Surface Acting on Managers'Fonctioning Through Psychological Needs » - Journal of occupational Health Psychology. DOI : $10.1037 /$ ocp 0000080

Joing I. \& Vors O. (2014) Le temps des vestiaires d'EPS au collège : climat, victimation et stratégies enseignantes. Congrès de l'ARIS : Genève.

Joing I. \& Vors O. (2015) «Victimation et climat scolaire au collège : les vestiaires d'Éducation Physique et Sportive »-Déviance et Société 39, $1(51-71)$.

Kong C. K. (2008) "Classroom learning experiences and students'perceptions of quality of school life »-Learning Environment Research 11 (111129).

Martin-Krumm C., Fenouillet F., Csillik A., Kern L., Besançon M., Heutte J., Paquet Y., Delas Y., Trousselard M., Lecorre B. \& Diener E. (2017) «Changes in Emotions from Childhood to Young Adulthood ». Child Indicators Research (1-21). Doi : 10.1007/s12187-016-9440-9

Ladd G. W., Kochenderfer B. J. \& Coleman C. C. (1996) « Friendship quality as a predictor of young children's early school adjustment »-Child Development $67(1103-1118)$.

Laguardia J.G. \& Ryan R.M. (2000) «Buts personnels, besoins psychologiques fondamentaux et bienêtre : théorie de l'autodétermination et applications »-Revue Québequoise de Psychologie 21, 2 (281- 304).

Lenoir M. (2012) Le bien-être de l'élève au college : representations des professeurs et des élèves. Université de Lyon 2 : thèse de doctorat.

Lester L. \& Cross D. (2015) «The Relationship Between School Climate and Mental and Emotional Wellbeing Over the Transition from Primary to Secondary School »-Psychology of Well-Being 5, 9. DOI : 10.1186/s13612-015-0037-8

Martensson F., Boldemann C., Söderström M., Blennow M., Englung J.-E. \& Grahn P. (2009) «Outdoor environmental assessment of attention promoting settings for preschool children »- Health \& Place 15, 4 (1149-1157).

Mawwell L. E. \& Chmielewski E. J. (2008) « Environmental personalization and elementary school children's self-esteem » - Journal of Environmental Psychology 28, 2 (143-153).

Mazalto M. \& Paltrinieri L. (2013) «Espaces scolaires et projets éducatifs » Revue Internationale d'Éducation, Sèvres 64 (31-40).

Mazalto M. (2017a) Concevoir des espaces scolaires pour le bien-être et la réussite. Paris : L'Harmattan. 
Mazalto M. (2017b) «Le bien-être dans les espaces scolaires » - Administration \& Éducation 156, 4 (29-34).

Myers D. G. (1992) The pursuit of happiness : Who is happy - and why. New York: William Morrow.

Nguyen T. P. (2016) La qualité de vie et le bien-être à l'école en France : quelle place dans les textes institutionnels? Rapport commandité par le Cnesco.

Noddings N. (2003) Happiness and education. Cambridge : Cambridge UP.

Pourchet G. \& Cerfontaine J. Y. (2004) « Des espaces d'enseignement aux lieux de vie : leçons d'une enquête »-Revue de l'Inspection Générale 2.

Reis H. T. (1994) "Domains of experience : Investigating relationship processes from three perspectives » - in : R. Erber and R. Gilmour (eds.) Theoretical frameworks for personal relationships (87-110). Hillsdale, NJ : Lawrence Erlbaum.

Reis H. T., Sheldon K., Gable S. \& Ryan R. M. (2000) "Daily Well-Being : The Role of Autonomy, Competence, and Relatedness » - Personality and Social Psychology Bulletin, 26, 4 (419-435).

Ryan R. M. (1995) «Psychological needs and the facilitation of integrative processes »- Journal of Personality 63 (397-427).

Ryan R. M. \& Deci E. L. (2000) « Self-determination theory and the facilitation of intrinsic motivation, social development, and well-being »-American Psychologist 55, 1 (68 - 78). DOI : 10.1037/0003-066X.55.1.68

Ryan R. M., \& Deci E. L. (2002) «Overview of self-determination theory : An organismic dialectical perspective » - in : E. L. Deci and R. M. Ryan (eds.) Handbook on self-determination research (3-33). Rochester, NY : University of Rochester Press.

Ryff C. D. (1995) « Psychological well-being in adult life »-Current Directions in spychological Science 4 (99-104).

Samdal O., Wold B. \& Bronis M. (1999) «Relationship between student's perceptions of school environment, their satisfaction with school and perceived academic achievement: An international study »- School Effectiveness and School Improvement 10 (296-320).

Sheldon K., \& Kasser T. (1995) «Coherence and congruence: Two aspects of personality integration »- Journal of Personality and Social Psychology 68 (531-543).

Sheldon K. M., Ryan R. M., Rawsthrne L. \& Ilardi B. (1997) «"True” self and "trait" self : Cross-role variation in the Big Five traits and its relations with authenticity and well-being » - Journal of Personality and Social Psychology 73 (1380-1393).

Sheldon K. M., Ryan R. M. \& Reis H. T. (1996) «What makes for a good day? Competence and autonomy in the day and in the person $»-$ Personality and Social Psychology Bulletin 22 (1270-1279).

Sgard A. \& Hoyaux, A. F. (2006) «L'élève et son lycée : de l'espace scolaire aux constructions des territoires lycéens »-L'information Géographique 70, 3 (87-108).

Tian L., Han M. \& Huebner E. S. (2014) « Preliminary development of the Adolescent Students' Basic Psychological Needs at School Scale »Journal of Adolescence 37, 3 (257-267).

Tian L., Tian Q \& Huebner E. S. (2015) « School-Related Social Support and Adolescents' School Related Subjective Well-Being: The Mediating 
Role of Basic Psychological Needs Satisfaction at School Grounded in Basic Psychological Needs Theory» - Social Indicators Research 11, 4 (227-268).

Tian L., Tian Q. \& Huebner E. S. (2016) «School-Related Social Support and Adolescents'School-Related Subjective Well-Being : The Mediating Role of Basic Psychological Needs Satisfaction at School »- Social Indicators Research 128, 1 (105-129).

Tomyn A. J. \& Cummins R. A. (2011) « The subjective well-being oh high school students: Validating the Personal Wellbeing Index-School Children » - Social Indicators Research 101 (405-418).

Verkuyten M. \& Thijs J. (2002) « School Satisfaction of Elementary School Children : The Role of Performance, Peer Relations, Ethnicity and Gender » - Social Indicators Research 59, 2 (203-228).

Véronneau M. H., Richard F., Koestner J. \& Abela R.Z. (2005) «Intrinsic Need Satisfaction and Well-Being in Children and Adolescents : An Application of the Self-Determination Theory » - Journal of Social and Clinical Psychology, 24, 2 (280-292).

Wachs S. (2012) «Moral disengagement and emotional and social difficulties in bullying and cyberbullying : Difference by participant role »-Emotional and Behavioural Difficulties 17 (347-360).

DOI : $10.1007 / \mathrm{s} 11482-012-9167-9$

Waterman A. S. (1993) «Two conceptions of happiness : Contrasts of personal expressiveness (eudaemonia) and hedonic enjoyment »-Journal of Personality and Social Psychology 64 (678-691).

Winterbottom M. \& Wilkins A. (2009) «Lighting and discomfort in the classroom »- Journal of Environmental Psychology 29, 1 (63-75). 